\title{
ABOUT THE POTENTIAL OF LIDARS WITH DIFFERENT PHOTODETECTORS UNDER DAYTIME SKY RADIATION Ravil Agishev ${ }^{1}$, Adolfo Comeron ${ }^{2}$, Alexander Gilerson ${ }^{3}$ \\ ${ }^{1}$ Kazan State Power Engineering University, 51, Krasnoselskaya St., Kazan 420066, Russia \\ ${ }^{2}$ Polytechnic University of Catalonia, 1-3, Jordi Girona, Barcelona 08034, Spain \\ ${ }^{3}$ City College of the City University of New York, 140 St. \& Convent Ave., New York, NY 10031, USA
}

\begin{abstract}
Results of theoretical analysis and experimental developments implemented as advanced methods and means to improve a noise-immunity of lidar systems for practical applications are discussed. A particular attention is paid to assessing the developed methods and technical solutions effectiveness and their comparison with existing lidar systems and real receivers.
\end{abstract}

\section{INTRODUCTION}

We discuss a renewed approach to the generalized methodology for atmospheric lidar assessment, which uses the dimensionless-parameterization as a core component [1-9]. It allows predicting its work under the presence of the sky background radiation using three different photodetectors - traditional PMTs, SiPMTs and APDs.

The renewed approach can be widely used to evaluate a broad range of lidar system capabilities for a variety of lidar remote sensing applications, as well as to serve as a basis for selection of appropriate lidar system parameters for a specific application.

\section{METHODOLOGY}

Such a modernized methodology provides generalized, uniform and objective approach for the evaluation of a broad range of lidar types and systems (aerosol, Raman, DIAL), operating on different targets (backscatter or topographic) and under intense sky background conditions. It can be used within the lidar community to compare different lidar instruments.

The signal to noise ratio at the lidar photodetector input $\Psi_{\mathrm{X}}$ can be decomposed as a product of five independent dimensionless parameters, each of which follows from a different source [8]:

$$
\Psi_{\mathrm{X}}=\mathrm{V} \mathrm{Qx} \mathrm{W} \mathrm{U}^{-1} \mathrm{r}^{-2}
$$

where $\mathrm{V}$ is the ratio of the echo-signal power $\mathrm{P}_{\text {sref }}$ received from the reference range $R_{\text {ref }}$ of the reference molecular atmosphere to the photo-detector threshold power $\mathrm{P}_{\mathrm{t} 0}$ in the absence of background noise; $\mathrm{Q}_{\mathrm{x}}$ is the backscatter efficiency of the arbitrary species to the molecular reference; $\mathrm{W}$ is the normalized atmospheric component determined by transparencies ratio of the current atmosphere state and the standard molecular atmosphere; $\mathrm{U}$ is the ratio of photodetector's threshold powers taken in presence and in absence of background radiation; $r$ is the normalized range-factor.

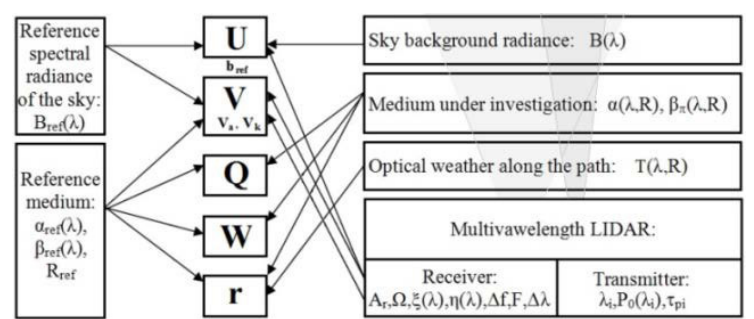

Fig.1. Schematic illustration of the parametrization principle.

power $\mathrm{P}_{\mathrm{t} 0}$ in the absence of background noise; $\mathrm{Q}_{\mathrm{x}}$ is the backscatter efficiency of the arbitrary species to the molecular reference; $\mathrm{W}$ is the normalized atmospheric component determined by transparencies ratio of the current atmosphere state and the standard molecular atmosphere; $U$ is the ratio of photodetector's threshold powers taken in presence and in absence of background radiation; $r$ is the normalized range-factor.

When analyzing the potential of any lidar system and in order to have a clear idea about the particular lidar system response at standard atmospheric conditions, in [9] it was proposed to adapt the optical parameters of sounded medium to parameters of the molecular atmosphere with pre-agreed parameters. This allows you to predict more effectively the behavior of lidar at certain atmospheric conditions.

\section{RESULTS}

As noted, the previously entered reference medium [1-9] has enabled to adapt the arbitrary optical "weather" to standardized parameters of molecular atmosphere. The modified universal lidar parame- 
ter for each sounding wavelength can be obtained by using the following three facts: (a) by introduction of standardized conditions of external environment's background radiation in the form of its brightness according to Eq. (2); (b) by taking into account the background brightness in Eq. (4) for threshold power of lidar signal; and (c) by subsequent joint account of reference environment and reference background brightness in analytical expressions for V-parameter calculations.

So when comparing different types of lidars it is useful to identify the $\mathrm{V}$-parameter as a universal dimensionless constant of multi-wavelength system at the specific wavelength $\lambda=\lambda_{i}$ (i.e. $V_{i}$-parameter at $\lambda_{\mathrm{i}}$ ) that characterizes the lidar's capability to remote sensing [7-9] and describes not only the energetic potential, but the lidar instrument's immunity to background radiation as well. Numerically, the V-parameter can be redefined [1] as a ratio of the echo-signal power $\mathrm{P}_{\text {srefi }}$ received from the reference range $R_{\text {ref }}$ of the reference atmosphere (for example, at molecular atmosphere conditions) to the photodetector's threshold power $\mathrm{P}_{\mathrm{tBref}}\left(\lambda_{\mathrm{i}}\right)$ at reference background conditions (for example, at conditions of «averaged» sky background brightness on the middle latitudes):

$$
\mathrm{V}_{\mathrm{i}} \equiv \mathrm{P}_{\mathrm{s} 0}\left(\lambda_{\mathrm{i}}\right) / \mathrm{P}_{\mathrm{tBref}}\left(\lambda_{\mathrm{i}}\right)=\sqrt{P R F \cdot T_{0}} \cdot P_{\text {stref }} / P_{t 0}
$$

The signal-to-noise ratio at the photodetector output of a lidar receiver channel can be presented in a simple and translucent form [9] with no pulse inte-gration (i.e. $\left(\mathrm{RFT} \times \mathrm{T}_{0}\right)^{1 / 2}=1$ ) as follow:

$$
\mathrm{SNR}=\mathrm{P}_{\mathrm{s}} /\left[\mathrm{P}_{\mathrm{q}}\left(\mathrm{P}_{\mathrm{s}}+\mathrm{P}_{\mathrm{b}}\right)+\mathrm{P}_{\mathrm{n}}^{2}\right]^{1 / 2}
$$

where $P_{s}$ and $P_{b}$ are respectively the optical signal power and the background-radiation power reaching the active surface of the photodetector. The parameter $P_{q}$, with power units, is given by

$$
\mathrm{P}_{\mathrm{q}}=2 \mathrm{~h} \mathrm{c} \mathrm{F} \Delta \mathrm{f} / \eta \lambda
$$

where $h$ is Planck's constant, $c$ is the speed of light, $F$ is the photodetector excess noise ratio, $\Delta f$

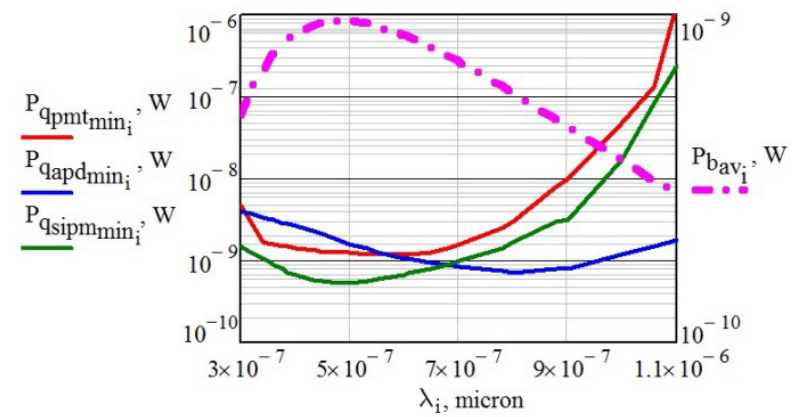

Fig. 2. Quantum noise and sky radiation power as the wavelength functions. is the photoreceiver's electrical bandwidth, $\eta$ is the photodetector's quantum efficiency, and $\lambda$ is the radiation wavelength.

In turn, the parameter $P_{n}$ is given by

$$
\mathrm{P}_{\mathrm{n}}=\mathrm{NEP} \cdot \Delta \mathrm{f}^{1 / 2}
$$

with $N E P$ the noise equivalent power of the photoreceiver.

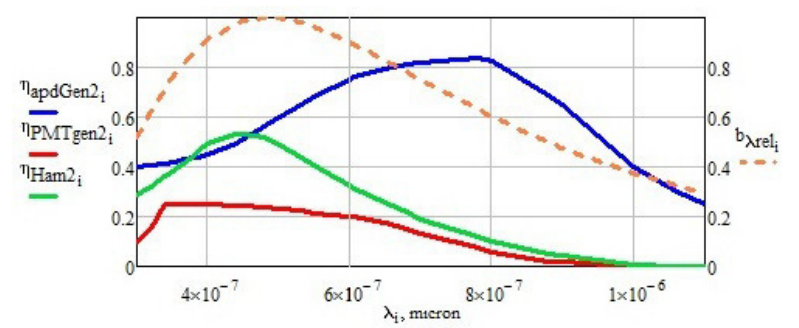

Fig. 3. Relations between three photodetectors quantum efficiency and the wavelength.

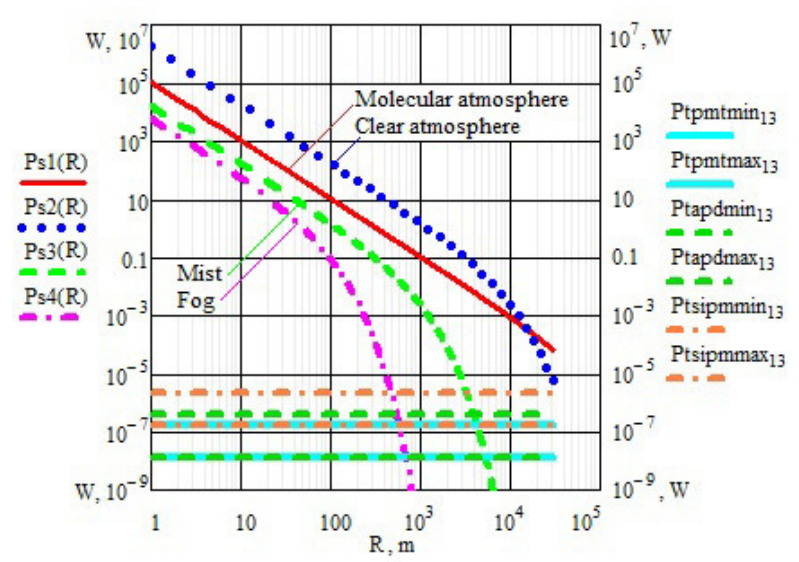

Fig. 4. Lidar returns and threshold signals of different photodetectors.

Under no background radiation conditions $\left(P_{b}=0\right)$ we will call the signal-to-noise ratio $S N R_{D}$, where the subscript D stands for "dark" as far as background radiation is concerned. A doubly logarithmic graph of as a function of can be approximated very well by two asymptotic straight lines, one of slope 1, given by [2]

$$
\mathrm{SNR}_{\mathrm{D}}=\mathrm{P}_{\mathrm{s}} / \mathrm{P}_{\mathrm{n}}
$$

for $\mathrm{P}_{\mathrm{s}}<\mathrm{P}_{\mathrm{n}}{ }^{2} / \mathrm{P}_{\mathrm{q}}$, and another of slope $1 / 2$, given by

for $\mathrm{P}_{\mathrm{s}}>\mathrm{P}_{\mathrm{n}}{ }^{2} / \mathrm{P}_{\mathrm{q}}$.

$$
\mathrm{SNR}_{\mathrm{D}}=\left(\mathrm{P}_{\mathrm{s}} / \mathrm{P}_{\mathrm{n}}\right)^{1 / 2}
$$

We can define the «knee» signal power at which the transition from slope 1 to slope $1 / 2$ occurs as

$$
\mathrm{P}_{\mathrm{sk}}=\mathrm{P}_{\mathrm{n}}^{2} / \mathrm{P}_{\mathrm{q}}
$$

Accepting the asymptotic approximation, which will overestimate the output signal-to-noise ratio in the neighborhood of the «knee», with a maximum overestimation of $3 \mathrm{~dB}$ just when $\mathrm{P}_{\mathrm{s}}=\mathrm{P}_{\text {sk }}$, the out- 
put $\mathrm{SNR}_{\text {out }}$ can be easily calculated as a function of the dimensionless parameters $\mathrm{V}_{\mathrm{a}}$ and $\mathrm{V}_{\mathrm{k}}$, and of the power decrease $\mathrm{V}^{\prime}$ [2] with respect to the reference input signal level $\mathrm{P}_{\text {sref }}$. Working in logarithmic units, if $\mathrm{V}_{\mathrm{k}}^{\prime}<\mathrm{V}_{\mathrm{k}}$ we have

$$
\mathrm{SNR}_{\mathrm{D}}=\rho_{0}+1 / 2\left(\mathrm{~V}_{\mathrm{a}}-\mathrm{V}^{\prime}\right)
$$

whereas if $\mathrm{V}_{\mathrm{k}}^{\prime}>\mathrm{V}_{\mathrm{k}}$ we will have

$$
\mathrm{SNR}_{\mathrm{D}}=\rho_{0}+1 / 2\left(\mathrm{~V}_{\mathrm{a}}-\mathrm{V}^{\prime}\right)+1 / 2\left(\mathrm{~V}_{\mathrm{k}}-\mathrm{V}^{\prime}\right)
$$

where $\rho_{0}$ is the reference signal-to-noise ratio.

Eqs. (9) and (10) can be merged [2] in a single one using Heaviside's step function $\mathrm{u}(\mathrm{x})$ :

$$
\mathrm{SNR}_{\mathrm{D}}=\rho_{0}+1 / 2\left(\mathrm{~V}_{\mathrm{a}}-\mathrm{V}^{\prime}\right)+1 / 2\left(\mathrm{~V}_{\mathrm{k}}-\mathrm{V}^{\prime}\right) \mathrm{u}\left(\mathrm{V}^{\prime}-\mathrm{V}_{\mathrm{k}}\right)
$$

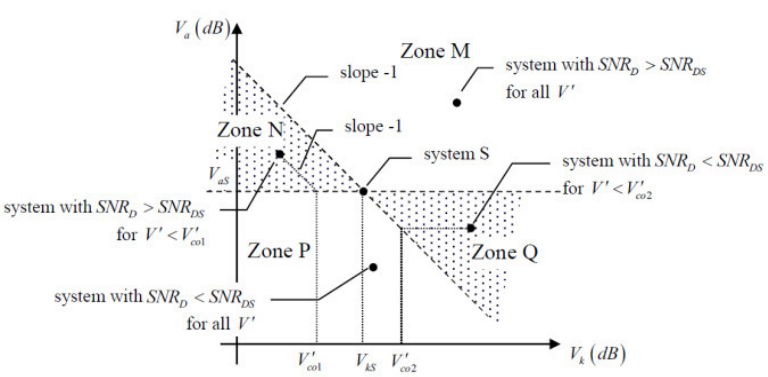

Fig. 5. Graphical comparison of systems.

The physical meaning of the earlier introduced and used U-parameter [1-9] is an extent of the lidar photo-detector's detection threshold degradation under intense daytime sky background.

$$
\begin{gathered}
U_{i} \equiv P_{t}^{B}\left(\lambda_{i}\right) / P_{t}^{\text {Bref }}\left(\lambda_{i}\right) \simeq \\
\simeq\left[1+\sqrt{1+\frac{4}{S N R_{\text {out }}^{2}} \frac{P_{b}\left(\lambda_{i}\right)}{P_{q}\left(\lambda_{i}\right)}}\right] /\left[1+\sqrt{1+\frac{4}{S N R_{\text {out }}^{2}} \frac{P_{\text {bref }}\left(\lambda_{i}\right)}{P_{q}\left(\lambda_{i}\right)}}\right]
\end{gathered}
$$

Since it is true for SiPM, PMT and APD detectors: $\mathrm{P}_{\mathrm{n}}<<\mathrm{P}_{\mathrm{q}}$, the formula (9) can be simplified. Then it is easy to see that under intense daytime sky background with $\mathrm{P}_{\mathrm{b}}>>\mathrm{P}_{\mathrm{q}}>>\mathrm{P}_{\mathrm{n}}$, the U-parameter as a degree of detection threshold increasing under the intense background radiation, for $\rho_{\text {out }}=1$ becomes as follows [1]:

$$
U_{i}\left(\lambda_{i}\right) \approx \sqrt{\frac{P_{b}\left(\lambda_{i}\right)}{P_{\text {bref }}\left(\lambda_{i}\right)}}
$$

Since we have chosen the reference background brightness in order to provide the reference background power to be the same order of magnitude as the photodetector's quantum noise, i.e. $\mathrm{P}_{\text {bref }} \sim \mathrm{P}_{\mathrm{q}}$, then Eq. (13) can be rewritten in a simpler form as follows:

$$
U_{i}=\frac{2 \sqrt{B_{\lambda i} A_{r} \xi\left(\lambda_{i}\right) \Omega \Delta \lambda \lambda_{i} \eta\left(\lambda_{i}\right) / \mathrm{hcF} \Delta f}}{1+\sqrt{1+2 B_{r e f \lambda_{i}} A_{r} \xi\left(\lambda_{i}\right) \Omega \Delta \lambda \lambda_{i} \eta\left(\lambda_{i}\right) / \mathrm{hcF} \Delta f}}
$$

where $B_{\lambda i}$ is the spectral radiance of the sky zone falling in the receiver field of view at $\lambda=\lambda_{i}$.
As we are interested to know what is the extent of operation range reduction under strong sky background for different detectors, by following [7] we can obtain the expression:

$r_{b} \equiv \frac{R_{B}}{R_{\text {ref }}}=\sqrt{\left[\sqrt{1+\frac{4}{\rho_{\text {out }}^{2}} \frac{P_{n}^{2}}{P_{q}^{2}}\left(1+\frac{P_{b} P_{q}}{P_{n}^{2}}\right)}-1\right] /\left[\left(1+\frac{P_{b} P_{q}}{P_{n}^{2}}\right)\left(\sqrt{1+\frac{4}{\rho_{\text {out }}^{2}} \frac{P_{n}^{2}}{P_{q}^{2}}}-1\right)\right]}$

If we apply (15) to lidars with different photodetectors, when inequalities $\mathrm{P}_{\mathrm{b}}>>\mathrm{P}_{\mathrm{q}}>>\mathrm{P}_{\mathrm{n}}$ are satisfied, and then use $\mathrm{U}_{\mathrm{i}}$ from (10), we can obtain:

$$
r_{b}=U_{i}^{-1 / 2}
$$

and for specific receiver parameters and certain sky background

$$
r_{b}=\sqrt[4]{2 h c \Delta f / \lambda} \cdot \sqrt[4]{F / \eta(\lambda)} / \sqrt[4]{P_{b}}
$$

By applying all the specific features of the lidar system and assessments necessary to the silicon photodetectors under consideration, we obtained results shown in Fig. 6.
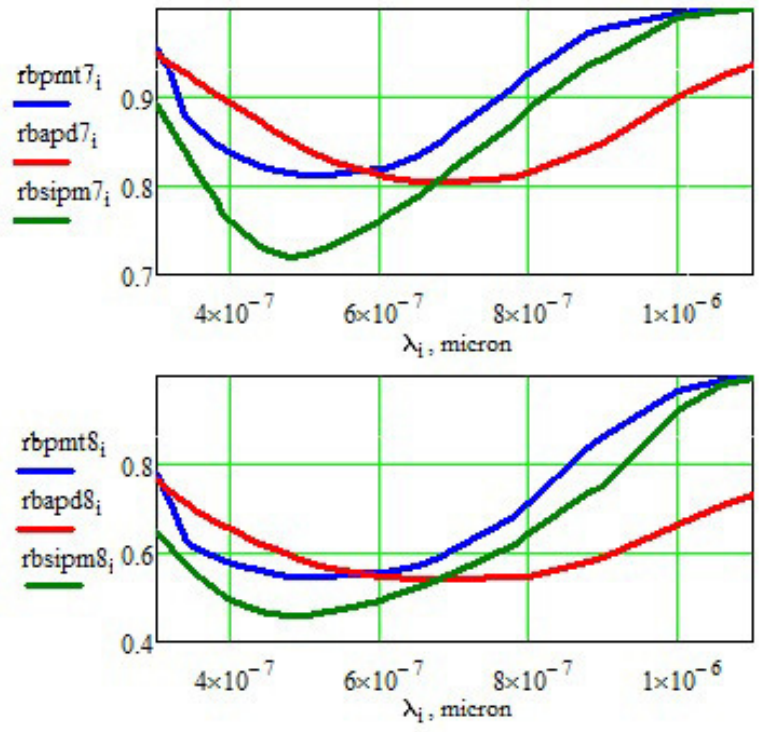

Fig. 6. $\mathrm{r}_{\mathrm{b}}$ as a function of $\lambda$ for $\mathrm{B}_{\lambda}=10^{7}$ and $\mathrm{B}_{\lambda}=10^{8}$ $\mathrm{W} \cdot \mathrm{m}^{-2} \mathrm{sr}^{-1} \mathrm{~m}^{-1}$ at $\lambda=483 \mathrm{~nm}$.

\section{CONCLUSIONS}

In the development of our previous approaches [1-9], we considered the practical applicability of the Vand $\mathrm{U}$ - parameters by extending it to all wavelengths of multi-wavelength sensing lidar and by adapting it to the reference level of the daytime sky back-ground on the basis of use of a more general approach to estimate the threshold power. Using the reference brightness of background radiation within its usual range of changes allows to evaluate the vulnerability of a particular system of impact of the background noise of moderate intensity and to 
predict reasonably the degree of deterioration of the lidar's detection threshold for the impact of sky background of arbitrary intensity.

These parameters allow predicting with a good approximation the signal-to-noise ratio at the photoreceiver output as a function of the signal variation with respect to a reference level. They also provide a quick way of comparing the performance of different systems.

\section{REFERENCES}

[1] R.Agishev, A.Comerón, A.Rodriguez, M.Sicard. Dimensionless parameterization of LIDAR for laser remote sensing of the atmosphere and its application to systems with SiPM and PMT detectors, Applied Optics, 2014, 53, № 15, 3164-3175.

[2] A.Comerón, R.Agishev. Dimensionless parameters for lidar performance characterization / in: Remote Sensing of Clouds and the Atmosphere XIX; and Optics in Atmospheric Propagation and Adaptive Systems XVII, SPIE Proceedings, 2014, 9242, pp.1-10.

[3] R.Agishev, A.Comerón, J.Bach, A.Rodriguez, M.Sicard, J.Riu, S.Royo. Lidar with SiPM: Some capabilities and limitations in real environment, Optics \& Laser Technology, 2013, 49, 86-90.

[4] R.Agishev. Introduction to SiPMs as novel and advanced detectors for Lidar remote sensing: Operation principles, main parameters, and state-ofthe-art. Analytical Review, UPC Publishing House, Barcelona, Spain, 2012, 115 pp.

[5] R.Agishev. Advanced methods and means to improve atmospheric lidar stability against sky background clutter, in: Remote sensing of clouds and the atmosphere, SPIE Proceedings, 2011, 8177, 177-183.

[6] R.Agishev. Lidar monitoring of the atmosphere: Monograph (in Russian), PhysMathGIz, Moscow, 2009. 316 pp.

[7] R.Agishev, B.Gross, F.Moshary, A.Gilerson, S.Ahmed. Simple approach to predict APD/PMT lidar detector performance under sky background using dimensionless parameterization, Optics and Lasers in Engineering, 2006, 44, No.8, 779-796.

[8] R.Agishev, B.Gross, F.Moshary, A.Gilerson, S.Ahmed, Development of a SNR parameterization scheme for general lidar assessment, Applied Phys- ics B: Lasers and Optics, 2005, vol. 80, No. 6, pp. 765-776.

[9] R.Agishev, A.Comeron, B.Gross, F.Moshary, S.Ahmed, A.Gilerson, V.Vlasov, Application of the method of decomposition of lidar signal-to-noise ratio to the assessment of laser instruments for gaseous pollution detection, Applied Physics B, 2004, 79(2), 255-264 (2004). 